\title{
Impact of Candida species on clinical outcomes in patients with suspected ventilator-associated pneumonia
}

\author{
Marie-Soleil Delisle MSc(Pharm) BCPS1 , David R Williamson MSc(Pharm) BCPS², Martin Albert MD FRCP ${ }^{3}$, \\ Marc M Perreault PharmD BCPS ${ }^{4}$, Xuran Jiang MSc ${ }^{5}$, Andrew G Day MSc ${ }^{5}$, Daren K Heyland MD MSc FRCP5,6
}

M-S Delisle, DR Williamson, M Albert, et al. Impact of Candida species on clinical outcomes in patients with suspected ventilatorassociated pneumonia. Can Respir J 2011;18(3):131-136.

BACKGROUND: The significance of Candida species in respiratory tract (RT) secretions in critically ill patients is unclear.

METHODS: A retrospective analysis of the Canadian ventilator-associated pneumonia (VAP) trial was conducted. Only patients with suspected VAP whose initial cultures failed to grow any known pathogens were included. Using two fundamentally different statistical techniques that adjusted for important confounding variables, the clinical outcomes of patients with Candida species recovered from RT cultures were compared with patients whose RT cultures were not positive for Candida species.

RESULTS: RT cultures yielded no identifiable bacterial pathogens in 274 patients; 68 patients had Candida species in the RT alone, while 206 patients did not have Candida species recovered from any site. The unadjusted OR of hospital mortality for patients with Candida species was 2.9 (95\% CI 1.6 to $5.2 ; \mathrm{P}<0.001)$. The hazard ratio of time to hospital discharge was 0.54 ( $95 \%$ CI 0.38 to $0.77 ; \mathrm{P}=0.001$ ). Logistic regression analysis demonstrated that age, Acute Physiology score and Chronic Health Evaluation (APACHE) II score, primary diagnosis of respiratory failure, two or more comorbidities and Candida species were independently associated with increased hospital mortality. Similar trends were observed with time to hospital discharge. The association between Candida species and increased mortality remained after controlling for potential confounders using both propensity score stratification and multivariable modelling approaches.

CONCLUSIONS: Patients with suspected VAP, in whom no bacterial pathogen was identified and in whom Candida species were isolated from RT cultures, exhibited a greater burden of illness compared with similar patients without Candida. Whether Candida species colonization of RT secretions is a marker of disease severity or actually contributes to poorer clinical outcomes remains unclear.

Key Words: Candida infection; Critical care; Intensive care; Nosocomial infection; Pneumonia

$\mathrm{V}$ entilator-associated pneumonia (VAP) is a common problem in intensive care unit (ICU) patients, with various studies reporting rates of $10 \%$ to $65 \%$ (1-9). In the recent Canadian VAP study (10), no bacterial pathogen was identified in $33.3 \%$ of enrollment cultures in patients with a clinical suspicion of VAP (CSVAP). Other studies of CSVAP demonstrated 'culture-negative' VAP rates of $7.5 \%$ to $54.6 \%$ (11-13). In a retrospective analysis of the Canadian VAP trial (14), patients with culture-negative CSVAP exhibited longer durations of mechanical ventilation (MV) and ICU stay, and higher ICU and hospital mortality rates compared with patients harbouring a bacterial pathogen identified in respiratory tract (RT) secretions. These observations suggest that the absence of a treatable bacterial pathogen may be associated with increased attributable morbidity and mortality compared with culture-positive VAP.
Les effets des espèces de Candida sur les issues cliniques des patients ayant une pneumonie qu'on présume être associée au respirateur

HISTORIQUE : On ne connaît pas la signification de la présence d'espèces de Candida dans les sécrétions des voies respiratoires (VR) de patients gravement malades.

MÉTHODOLOGIE : Les chercheurs ont procédé à l'analyse rétrospective d'un essai canadien sur les pneumonies associées au respirateur (PAR). Seuls les patients ayant une PAR présumée dont la première culture ne comportait aucun pathogène connu y participaient. Au moyen de deux techniques statistiques fondamentalement différentes, rajustées pour tenir compte de variables confusionnelles importantes, ils ont comparé les issues cliniques de patients chez qui des espèces de Candida avaient été prélevées dans les cultures des VR à celles de patients dont les cultures des VR n'étaient pas positives aux espèces de Candida.

RÉSULTATS : Les cultures des VR n'ont produit aucun pathogène bactérien identifiable chez 274 patients; 68 patients présentaient des espèces de Candida seulement dans les VR, tandis que chez 206 patients, aucune espèce de Candida n'avait été prélevée dans l'un ou l'autre des foyers. Le risque relatif non rajusté de mortalité hospitalière chez les patients présentant des espèces de Candida était de 2,9 (95 \% IC 1,6 à 5,2; P<0,001). Le rapport de risque du délai jusqu'au congé hospitalier correspondait à 0,54 (95\% IC 0,38 à 0,77; P=0,001). L'analyse de régression logistique a démontré que l'âge, l'indice de physiologie aiguë et l'évaluation de santé chronique (APACHE), le diagnostic primaire d'insuffisance respiratoire, deux comorbidités ou plus et les espèces de Candida étaient indépendamment associées à une mortalité hospitalière accrue. Les chercheurs ont observé des tendances similaires du délai jusqu'au congé hospitalier. L'association entre les espèces de Candida et une mortalité accrue est demeurée après avoir contrôlé les variables confusionnelles potentielles au moyen de la stratification des indices de propension et des démarches de modélisation multivariables.

CONCLUSIONS : Les patients chez qui on présume une PAR, chez qui on n'a repéré aucun pathogène et chez qui les espèces de Candida ont été isolées des cultures des VR ont présenté un plus grand fardeau de maladie que les patients similaires sans Candida. On ne sait pas si les colonisations des espèces de Candida dans les sécrétions des VR sont un marqueur de gravité de la maladie ou contribuent plutôt à de moins bonnes issues cliniques.

Candida species are frequently recovered from RT secretions in mechanically ventilated nonimmunocompromised ICU patients, reaching $75.3 \%$ in a cohort of patients staying a median of 17 days in the ICU (15). Historically, these have been considered not to be pathogenic (16-22); however, recent studies have questioned this assumption (23). In a secondary analysis of the Canadian VAP trial (24), we found a significant association between the presence of Candida species in the RT and hospital mortality.

We hypothesized that the presence of Candida species in RT secretions may explain the excess morbidity and mortality seen in patients with culture-negative VAP. Thus, the primary objective of the present study was to compare hospital mortality and time to discharge from hospital between patients with and without the presence of Candida species in RT culture specimen in patients with CSVAP in which no

${ }^{1}$ Department of Pharmacy Services, The Montreal General Hospital; ${ }^{2}$ Department of Pharmacy Services, Hôpital du Sacré-Coeur de Montréal, Faculté de Pharmacie, Université de Montréal; ${ }^{3}$ Departments of Critical Care and Internal Medicine, Hôpital du Sacré-Cour de Montréal, Faculté de Médecine; ${ }^{4}$ Department of Pharmacy Services, The Montreal General Hospital and Faculté de Pharmacie, Université de Montréal, Montréal, Quebec; ${ }^{5}$ Clinical Evaluation Research Unit, Kingston General Hospital; ${ }^{6}$ Department of Medicine, Queen's University, Kingston, Ontario

Correspondence: Dr Daren K Heyland, Kingston General Hospital, Angada 4, Kingston, Ontario K7L 2V7. Telephone 613-549-6666 ext 3339, fax613-548-2428, e-mail dkh2@queensu.ca 
bacterial pathogen was isolated. We performed a multivariable analysis and a propensity analysis to control for potential confounding variables to determine the overall effect of Candida species in the lung on clinical outcomes.

\section{METHODS}

\section{Design}

A retrospective exploratory analysis was conducted on a subset of patients from the Canadian randomized, multicentre VAP trial (10). The complete study design and results of the trial have been published elsewhere (25).

\section{Population}

Adult patients were included in the VAP trial if they were admitted to an ICU for longer than $96 \mathrm{~h}$, were mechanically ventilated for longer than $48 \mathrm{~h}$ and if they fulfilled criteria for a CSVAP, which had to be met within $48 \mathrm{~h}$ of enrollment. Immunocompromised patients, defined as postorgan transplantation, HIV or neutropenic (less than 1000 absolute neutrophils) patients, or those receiving corticosteroids (more than $20 \mathrm{mg} /$ day of prednisone or equivalent) for more than six months, were excluded, as were patients infected or colonized with Pseudomonas species or methicillin-resistant Staphylococcus aureus (refer to reference 10 for the complete list of exclusion criteria and definitions of CSVAP). Enrolled patients were randomly assigned to either endotracheal aspiration or bronchoaveolar lavage, and either monotherapy or combination therapy.

Patients enrolled in the present VAP study were identified retrospectively, and harboured no identifiable bacterial pathogens in their RT secretions on enrollment day. Patients with known bacterial pathogens were excluded. Coagulase-negative Staphylococci, Bacteroides species and Clostridium species were not considered pathogens in RT secretions. Patients with 'mixed flora' or 'common flora' were also included. Subjects were divided in two groups according to whether Candida species were retrieved from any RT culture (endotracheal aspirate or bronchoalveolar lavage) on enrollment day (Candida group) or not (non-Candida group). Patients with positive Candida species culture from any site other than the RT on enrollment day or positive Candida species culture from any site in the seven-day period before enrollment were excluded.

\section{Data collection}

Demographic data were collected on enrollment in the VAP trial. Culture results from all sites and antibiotic use for the seven days before enrollment and throughout the study period were recorded. The outcome measures of hospital length of stay and mortality were recorded.

\section{Statistical analysis}

Hospital mortality and time to discharge alive from hospital were defined a priori as the two primary outcomes of the present analysis. The association between these outcomes and the presence of Candida species was assessed before adjustment for baseline characteristics, and after adjustment by both the traditional multivariable modelling approach and the propensity score method. While traditional multivariable modelling reduced confounding bias by controlling for variables associated with outcome, the propensity score approach reduced confounding bias by balancing variables associated with Candida colonization. Statistical analysis was performed using SAS version 9.1 (SAS Institute Inc, USA).

Categorical variables were described as counts and percentages and compared using the $\chi^{2}$ test, except for the mortality variables, which were compared between Candida groups by unadjusted ORs using Fisher's exact test. Time from randomization to MV discontinuation, ICU discharge and hospital discharge were described by Kaplan-Meier estimates of the median and quartiles. The hazard ratio of these events was estimated by the Cox proportional hazards model and was tested by the log-rank test. Patients who died before or within $24 \mathrm{~h}$ of these events were considered to have never successfully reached them and, were thus, censored after the end of follow-up. This censoring strategy produced essentially the same estimates as treating death as a competing risk. Other continuous variables were described as either means with SDs and compared using the $t$ test or, if they were substantially skewed, as medians with quartiles and compared using the WilcoxonMann-Whitney test.

Logistic regression and the Cox proportional hazards model were used to compare the primary outcomes between Candida groups after adjusting for all baseline variables independently associated with mortality at $\mathrm{P}<0.1$ (Table 1 ). For continuous predictors, the linear term was augmented with two nonlinear terms as defined by a restricted cubic spline with three knots if the addition of the nonlinear terms significantly (ie, $\mathrm{P}<0.1$ ) improved the model fit $(26)$. This approach ensured that confounding variables were adequately controlled for even if they were not linearly related to the outcome. Furthermore, interactions between Candida groups and all baseline variables were tested to ensure adequate control in the presence of effect modification. All covariates, including nonlinear and interaction terms, were offered as candidate covariates to stepwise selection with an entry and exit criteria of $\mathrm{P}<0.1$. The area under the ROC curve (AUC) of 0.86 and the Hosmer-Lemeshow test $(\mathrm{P}=0.10)$ suggested a good fit of the logistic model (27). The proportional hazards assumption of the Cox proportional hazards model was confirmed by examining the log-log survival curves and the Schoenfeld residuals, and by testing for a time-dependent interaction between Candida and the log of time (28).

Given the relatively small sample size and extensive list of baseline covariates, it was decided a priori to verify the previous analyses using the propensity score approach because this approach does not rely on the number of events being large compared with the number of covariates (29). The probability of being in the Candida group (propensity score) was estimated by a logistic regression model including all baseline characteristics (12 parameters), their first-order interactions (seven parameters) and nonlinear terms (two parameters) retained by backward stepwise selection with entry and exit criteria of $\mathrm{P}<0.2$. This propensity model achieved an area under the ROC curve of 0.85 and the Hosmer-Lemeshow test $(\mathrm{P}=0.77)$, thereby suggesting a good fit to the data. Seventy-four patients with a propensity score below 0.042 were excluded (all non-Candida) as were the eight patients with a propensity score above 0.9 (all Candida) because at these extreme ranges of propensity, there were no comparable patients from the other groups. Thus, the propensity score analysis was based on 192 patients, of whom 53 (28\%) died in hospital. Included patients were then stratified into quintiles based on their propensity score such that the $20 \%$ of patients with the lowest probability of having Candida species according to their covariate profile were stratified to quintile 1 , and the $20 \%$ with the highest probability of having Candida species were stratified to quintile 5. The first and second quintiles were collapsed into a single stratum due to the paucity of Candida patients (five and seven, respectively) and the equivalence of mortality rates in these quintiles. Baseline patient characteristics were subsequently confirmed to ensure that they were well balanced between Candida groups within each quintile and overall after stratifying according to quintile. Thus, the analysis was stratified according to propensity quintile to eliminate confounding due to group differences in baseline covariates. Hospital mortality and time to hospital discharge were compared between Candida groups using the Mantel-Haenszel test and Cox proportional hazards model, and each were stratified according to propensity quintile. No interaction (effect modification) between the Candida effect and the propensity quintile was confirmed using the the Breslow-Day test for hospital mortality and by a likelihood ratio test for the time to hospital discharge. Finally, the multivariable logistic regression and Cox proportional hazards models were re-estimated after stratifying for (conditioning on) propensity quintile. 
TABLE 1

Baseline characteristics of patients with and without Candida species in respiratory tract secretions among patients with negative bacterial enrollment cultures

\begin{tabular}{l} 
Baseline characteristics \\
\hline Age, years \\
Sex, male, $n(\%)$ \\
Comorbidities, $n$ \\
Number of comorbidities, n (\%) \\
0 \\
1 \\
2 \\
$\geq 3$
\end{tabular}

Primary admission diagnosis, $\mathrm{n}(\%)$

Neurological disease

Sepsis

Trauma

Respiratory failure

Cardiovascular

Gastrointestinal

Other

Admission category, $\mathrm{n}(\%)$

Medical

Surgical

Duration of MV before

randomization, days

(median [Q1, Q3])

Duration of ICU stay before

randomization, days

(median [Q1, Q3])

At least one positive respiratory

culture in previous 7 days, $n$ (\%)

Antibiotic exposure in previous

3 days, $\mathrm{n}(\%)$

APACHE II score

(median [Q1, Q3]) ${ }^{\dagger}$

MOD score (median [Q1, Q3]) ${ }^{\dagger}$

CPIS (median [Q1, Q3])

CPIS $\geq 5$, n (\%)

Individual signs of pneumonia

Temperature, ${ }^{\circ} \mathrm{C}$

Use of inotropes or

vasopressors, $\mathrm{n}(\%)$

Respiratory rate

$\mathrm{PaO}_{2} / \mathrm{FiO}_{2}$

White blood cells, $\times 10^{9} / \mathrm{L}$

Volume of endotracheal

secretions, $\mathrm{n}(\%)$

\section{Copious or large amount}

Moderate amount

Small amount or none

Purulence of endotracheal

secretions, $n$ (\%)

Purulent

Mucopurulent or mucoid

None or clear

Chest radiography results, $\mathrm{n}(\%)$

New infiltrate

Worsening or persistent infiltrate

Non-

Candida Candida Stratified

\begin{tabular}{cccc}
$(\mathbf{n}=68)$ & $(\mathbf{n}=\mathbf{2 0 6})$ & $\mathbf{P}$ & $\mathbf{P}$ \\
\hline $60.1 \pm 16.3$ & $60.4 \pm 17.9$ & 0.88 & 0.13
\end{tabular}

$40(58.8) \quad 144(69.9) \quad 0.09 \quad 0.83$

$\begin{array}{llll}1.6 \pm 1.1 & 1.5 \pm 1.1 & 0.67 & 0.39\end{array}$

$\begin{array}{lll}0.67 & 0.79\end{array}$

15 (22.1) 51 (24.8)

$17(25.0) \quad 55(26.7)$

$17(25.0) \quad 42(20.4)$

$19(27.9) \quad 58(28.2)$

$5(7.4) \quad 24(11.7)$

$4(5.9) \quad 9(4.4)$

$12(17.6) \quad 46(22.3)$

$20(29.4) \quad 44(21.4)$

$18(26.5) \quad 47(22.8)$

$6(8.8) \quad 20(9.7)$

$3(4.4) \quad 16(7.8)$

0.60

0.73

$44(64.7) \quad 126(61.2)$

$24(35.3) \quad 80(38.8)$

$6.7(4.6,9.4) \quad 6.0(4.8,8.1) \quad 0.48 \quad 0.36$

$6.9(5.1,10.8) 6.0(4.8,7.8) \quad 0.07 \quad 0.31$

$11(16.2) \quad 50(24.3) \quad 0.16 \quad 0.28$

$62(91.2) \quad 143(69.4)<0.001 \quad 0.70$

$\begin{array}{cccc}21.0 & 20.0 & 0.96 & 0.57 \\ (16.0,25.0) & (16.0,25.0) & & \\ 6.0(4.0,8.0) & 5.0(4.0,7.0) & 0.32 & 0.85 \\ 5.0(3.5,5.0) & 4.0(3.0,5.0) & 0.31 & 0.82 \\ 43(63.2) & 99(48.1) & 0.03 & 0.96 \\ & & & \\ 37.9 \pm 0.7 & 37.8 \pm 0.8 & 0.58 & 0.67 \\ 25(36.8) & 38(18.4) & 0.002 & 0.80 \\ & & & \\ 19.7 \pm 6.9 & 20.5 \pm 7.7 & 0.42 & 0.92 \\ 197.8 \pm 77.8 & 209.9 \pm 84.4 & 0.31 & 0.97 \\ 15.9 \pm 7.4 & 13.1 \pm 5.4 & 0.001 & 0.79 \\ & & 0.23 & 0.96\end{array}$

$12(17.6) \quad 58(28.2)$

$31(45.6) \quad 83(40.3)$

$25(36.8) \quad 65(31.6)$

$0.70 \quad 0.82$

$6(8.8) \quad 26(12.6)$

$57(83.8) \quad 165(80.1)$

$5(7.4) \quad 15(7.3)$

$0.06 \quad 0.99$

$11(16.2) \quad 57(27.7)$

57 (83.8) $149(72.3)$

Data presented as mean \pm SD unless otherwise indicated. *Stratified according to propensity quintile using sample of 60 Candida and 132 non-Candida patients with propensity scores of between 0.042 and $0.9 ;{ }^{\dagger}$ Reported Acute Physiology and Chronic Health Evaluation (APACHE) II and Multiple Organ Dysfunction (MOD) scores were calculated on day of enrollment. CPIS Clinical Pulmonary Infection Score; $\mathrm{FiO}_{2}$ Fraction of inspired oxygen; ICU Intensive care unit; MV Mechanical ventilation; $\mathrm{PaO}_{2}$ Partial pressure of arterial oxygen; Q1, Q3 Quintiles 1 and 3

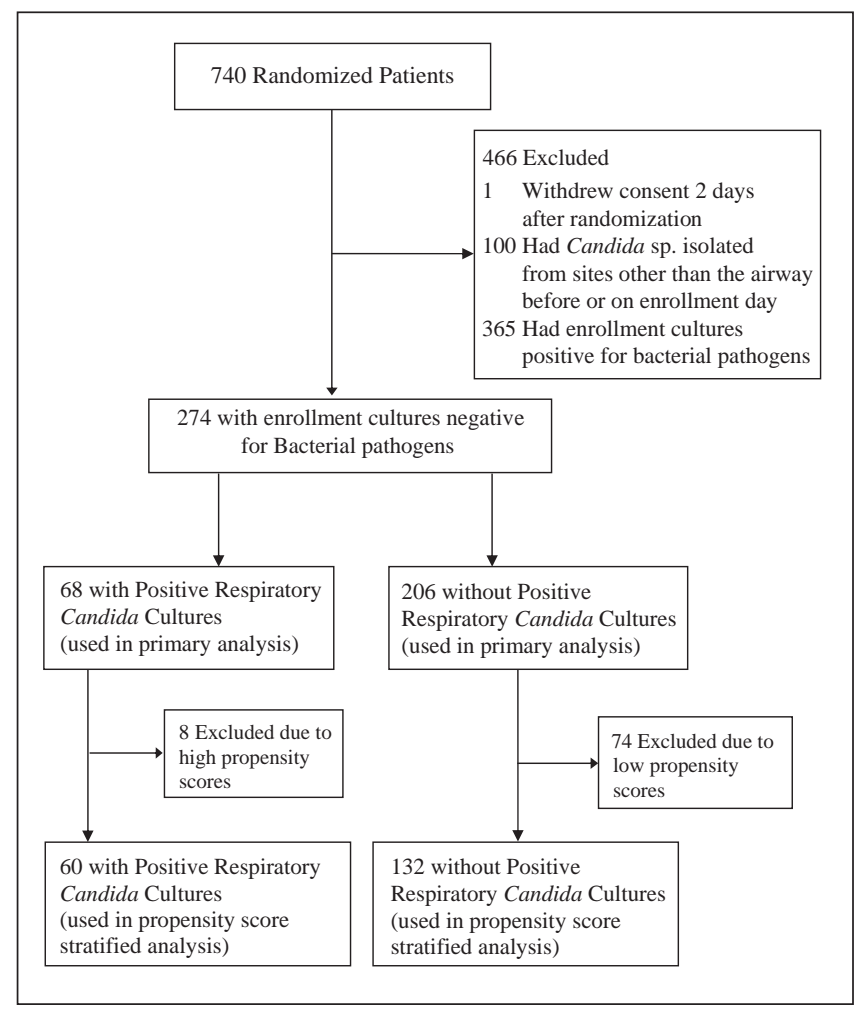

Figure 1) Patient flowchart. sp Species

\section{RESULTS}

The VAP study included 740 patients, with exclusions detailed in Figure 1. The final cohort for the present analysis consisted of 274 patients with negative bacterial enrollment cultures: 68 patients had Candida species confined to the RT alone, while 206 patients were included in the non-Candida group.

Patients with and without Candida species in RT secretions are compared in Table 1. Antibiotic exposure in the three days before enrollment was more frequent in the Candida group (91.2\% versus $69.4 \% ; \mathrm{P}<0.001)$. A greater proportion of Candida patients required vasopressors or inotropes $(36.8 \%$ versus $18.4 \% ; \mathrm{P}=0.002)$ and had a clinical pulmonary infection score of 5 or higher $(63.2 \%$ versus $48.1 \%$; $\mathrm{P}=0.03$ ). In the unadjusted analysis, patients with Candida species experienced significantly higher mortality rates (Table 2 ) and longer times from study enrollment to extubation, ICU and hospital discharge (Table 3 ).

In the adjusted analysis, age, Acute Physiology and Chronic Health Evaluation II score, primary diagnosis of respiratory failure, two or more comorbidities and Candida species were independently associated with increased hospital mortality (Table 4). A significant synergistic interaction between Candida species and two or more comorbidities was identified $(\mathrm{P}=0.035)$ such that the association between Candida and mortality was primarily observed in patients with two or more comorbidities (Table 4). The same signals were observed with time to hospital discharge (Table 5).

The above observations were confirmed using a propensity score approach to simultaneously balance all baseline patient characteristics between the Candida and non-Candida groups. Patient characteristics were well balanced between Candida groups within propensity strata and, after propensity stratification, no significant differences in baseline characteristics remained between patients with and without Candida species (adjusted $\mathrm{P}$ value in Table 1).

The OR of hospital mortality for Candida species in the lung, after stratifying for propensity quintile, was 2.5 (95\% CI 1.2 to 5.1; $\mathrm{P}=0.0093$ ) while the stratified hazard ratio for time to hospital 
TABLE 2

Mortality

\begin{tabular}{lcccc}
\hline & $\begin{array}{c}\text { Candida } \\
(\mathbf{n}=\mathbf{6 8})\end{array}$ & $\begin{array}{c}\text { Non-Candida } \\
(\mathbf{n}=\mathbf{2 0 6})\end{array}$ & $\begin{array}{c}\text { Candidal } \\
\text { non-Candida, } \\
\text { OR (95\% Cl) }\end{array}$ & $\mathbf{P}^{*}$ \\
\hline Died within 14 days & $15(22.1)$ & $21(10.2)$ & $2.49(1.20-5.17)$ & 0.02 \\
Died within 28 days & $21(30.9)$ & $31(15.0)$ & $2.52(1.33-4.79)$ & 0.007 \\
Died in ICU & $20(29.4)$ & $28(13.6)$ & $2.65(1.37-5.11)$ & 0.005 \\
Died in hospital & $29(42.6)$ & $42(20.4)$ & $2.90(1.61-5.23)$ & $<0.001$ \\
\hline
\end{tabular}

Data presented as $n$ (\%) unless otherwise indicated. *Unadjusted $P$ values

(Fisher's exact test). ICU Intensive care unit

\section{TABLE 3}

Duration of stay after randomization

\begin{tabular}{lcccc}
\hline & $\begin{array}{c}\text { Candida } \\
(\mathbf{n}=68)\end{array}$ & $\begin{array}{c}\text { Non-Candida } \\
(\mathbf{n}=\mathbf{2 0 6})\end{array}$ & $\begin{array}{c}\text { Candidal } \\
\text { non-Candida, } \\
\text { HR }(\mathbf{9 5 \%} \mathbf{C l})\end{array}$ & $\mathbf{P}^{*}$ \\
\hline $\begin{array}{c}\text { Time on } \\
\text { Ventilator }\end{array}$ & $13.0(4.3-\text { Und })^{\dagger}$ & $8.7(3.9-18.9)$ & $0.66(0.48-0.91)$ & 0.01 \\
ICU LOS & $17.3(7.1-$ Und $)$ & $13.0(6.9-27.3)$ & $0.68(0.49-0.94)$ & 0.02 \\
Hospital LOS 90.0 (27.8- Und) & $36.0(21.0-89.8)$ & $0.54(0.38-0.77)$ & 0.0005
\end{tabular}

Data presented as median (interquartile range) unless otherwise indicated. *Unadjusted values from log-rank test. ${ }^{\dagger}$ The upper quartile is undefined because less than $75 \%$ of patients ever experienced the event. ICU Intensive care unit; LOS Length of stay; Und Undefined

\begin{tabular}{|c|c|c|}
\hline & OR $(95 \% \mathrm{Cl})$ & $\mathbf{P}$ \\
\hline Age (per decade) & $1.96(1.42-2.70)$ & $<0.0001$ \\
\hline APACHE II on day of enrollment (per point) & $1.06(1.00-1.12)$ & 0.047 \\
\hline \multicolumn{3}{|l|}{ Primary diagnosis } \\
\hline Trauma (versus all other diagnoses) & $0.22(0.05-1.05)$ & 0.058 \\
\hline Respiratory failure (versus all other diagnoses) & $2.42(1.17-5.01)$ & 0.018 \\
\hline \multicolumn{3}{|l|}{ Number of comorbidities $\geq 2$ versus $\leq 1$} \\
\hline Patients without Candida & $1.62(0.68-3.84)$ & 0.27 \\
\hline Patients with Candida & $8.92(2.39-33.2)$ & 0.001 \\
\hline \multicolumn{3}{|l|}{ Enrollment respiratory Candida versus no Candida } \\
\hline Patients with $\leq 1$ comorbidity & $1.34(0.38-4.73)$ & 0.65 \\
\hline Patients with $\geq 2$ comorbidities & $7.40(2.83-19.3)$ & $<0.001$ \\
\hline $\begin{array}{l}\text { Interaction between Candida and } \geq 2 \\
\text { comorbidities }\end{array}$ & $5.50(1.23-26.9)$ & 0.035 \\
\hline
\end{tabular}

*This model includes all baseline characteristics (see Table 1) and their interactions with Candida that were retained by stepwise selection using $P<0.1$ as a criterion for entry and removal. The shaded rows are estimated from the model, but do not require additional parameters. Homer-Lemeshow goodness-of-fit test $P=0.10$ and model area under the ROC curve $=0.86$, which suggests adequate fit. APACHE II Acute Physiology and Chronic Health Evaluation II

discharge was 0.58 (95\% CI 0.38 to $0.88 ; \mathrm{P}=0.011$ ). There was no significant evidence that the $\mathrm{OR}$ or $\mathrm{HR}$ varied substantially between propensity quintiles (interactions $\mathrm{P}=0.80$ and $\mathrm{P}=0.91$, respectively). The conclusions from the multivariable analysis remained unchanged after stratification for propensity score, with only trivial modifications in the logistic regression and Cox proportional hazards estimates (data not shown).

No patient developed candidemia, and only three patients in the Candida group (4.4\%) and nine in the non-Candida group $(4.4 \%)$ grew Pseudomonas from RT culture specimens $48 \mathrm{~h}$ after enrollment. Fifteen patients $(22.0 \%)$ in the Candida group received intravenous antifungal treatment (mean duration 7.5 days [range 1 to 18 days]) compared with 26 patients $(12.6 \%)$ in the non-Candida group (mean duration 9.3 days [range one to 25 days]).
TABLE 5

Cox proportional hazards model of time to hospital discharge with interactions*

\begin{tabular}{lcc}
\hline & HR (95\% Cl) & P \\
\hline Age (per decade) & $0.89(0.81-0.97)$ & 0.009 \\
APACHE II on day of enrollment (per point) & $0.96(0.93-0.98)$ & 0.001 \\
Primary diagnosis of respiratory failure & $0.58(0.39-0.86)$ & 0.005 \\
$\quad$ (versus all other diagnoses) & & \\
Number of comorbidities ( $\geq 2$ versus $\leq 1)$ & $0.84(0.58-1.21)$ & 0.35 \\
Patients without Candida & $0.27(0.13-0.55)$ & $<0.001$ \\
Patients with Candida & $0.91(0.59-1.40)$ & 0.67 \\
Enrollment respiratory Candida (versus no Candida) & $<0.001$ \\
$\quad$ Patients with $\leq 1$ comorbidity & $0.29(0.15-0.55)$ & $<.004$ \\
Patients with $\geq 2$ comorbidities & $0.32(0.15-0.69)$ & 0.004 \\
Interaction between Candida and & & \\
$\geq 2$ comorbidities & & \\
\hline
\end{tabular}

*This model includes all baseline characteristics (see Table 1) and their interactions with Candida that were retained by stepwise selection using $P<0.1$ as a criterion for entry and removal. The shaded rows are estimated from the model, but do not require additional parameters. APACHE II Acute Physiology and Chronic Health Evaluation II

\section{DISCUSSION}

We conducted a retrospective analysis of patients from the Canadian randomized, multicentre VAP trial to assess whether isolated Candida species in the RT secretions of patients with negative bacterial enrollment cultures were associated with poorer patient outcomes (10). The major findings were that isolated Candida species from the RT secretions in this patient population were associated with higher ICU and hospital mortality, longer duration of MV use and ICU and hospital length of stay. These findings were robust to various statistical strategies that adjusted for the influence of confounding variables.

Previous studies (30) have suggested an association between Candida species colonization from various sites and prolonged ICU and hospital stay, and increased health care costs. Azoulay et al (23) observed that patients with Candida species colonization in the RT culture specimen specifically experienced a significantly longer period of MV, ICU and hospital stay compared with noncolonized patients. However, extra pulmonary colonization was documented in $39.7 \%$ of patients in that study, which raises the issue of different findings depending on patient populations and the degree of extrapulmonary colonization. In a previous analysis (24), we showed that isolated Candida colonization of RT secretions was associated with increased hospital mortality and length of stay, regardless of bacterial enrollment culture results. The current analysis focused on patients with isolated Candida species colonization of RT secretions, but with negative bacterial enrollment culture. Evidence is emerging that this specific patient population exhibits less favourable clinical outcomes compared with similar patients with a CSVAP but positive bacterial enrollment cultures (14). Our study is the first to investigate clinical outcomes in this specific population while accounting for the presence or absence of Candida species colonization of RT secretions.

There are two possible explanations for the described association between Candida and increased mortality. First, Candida species may modify patient outcomes by directly causing increased morbidity and mortality. In a postmortem histological study of non-neutropenic, mechanically ventilated ICU patients, el-Ebiary et al (19) reported a Candida species colonization rate of $40 \%$ (10 of 25 patients) and a Candida pneumonia incidence rate of $8 \%$ (two of 25 patients). In a similar study, Meersseman et al (21) reported that $57 \%$ of patients with pneumonia at autopsy had positive RT cultures for Candida premortem, but no Candida pneumonia was observed in autopsy specimens. We postulate that Candida species in the lung may still cause worse clinical outcomes in the absence of developing pneumonia. B-glucan is the major component of the yeast cell wall and has the potential 
to stimulate the production of pulmonary inflammation and cause cellular immune dysfunction $(31,32)$. In critically ill patients with suspected VAP and Candida species isolated from the RT secretions, we previously showed that such patients have levels of C-reactive protein, procalcitonin and interleukin- 6 that are comparable with patients experiencing bacterial infections (33). Thus, Candida species may be causally related to the increased morbidity and mortality described in the present study. Alternatively, Candida species-colonized patients may have physiological derangements that create a favourable environment for Candida overgrowth. These patients could have some degree of immunodeficiency for which Candida is a marker. Candida could then either be a marker of this immune dysfunction or directly play a role by causing or facilitating other coinfections. The recently reported interaction between Pseudomonas and Candida would support the latter theory $(23,34)$. The interaction between the presence of Candida species, the presence of comorbidities and mortality suggests that the former theory may be correct. In reality, a randomized trial of antifungal therapy in this specific population is needed to confirm this relationship. Such a trial is underway in Canada, conducted under the auspices of the Canadian Critical Care Trials Group (www.clinicaltrials.gov, number: NCT00934934).

The strength of the present study lies in its use of two statistical approaches - multivariable regression models and propensity scores to evaluate the relationship between Candida and various clinical outcomes, with both showing similar results. Other strengths include the large number of patients enrolled, data collection in a prospective, multicentre, randomized controlled setting and protocolization of airway specimen collection.

There are, however, also several limitations to the current analysis, one of them being that surveillance cultures of all other sites were not protocolized in the course of the VAP trial to ensure that patients were truly only colonized in the RT. Because it has been suggested that multifocal versus unifocal Candida colonization predicts a greater risk of candidal infection in ICU patients, it is a confounder that cannot be controlled (35). Another limitation was the assumption that Candida species recovered from RT secretions indicates colonization and not infection, although this is in concordance with other studies on the subject $(17,23,30)$. Data regarding central venous catheters and parenteral nutrition were not collected during the original trial. All laboratories did not speciate isolates of Candida; thus, complete data were

\section{REFERENCES}

1. Cook DJ, Kollef MH. Risk factors for ICU-acquired pneumonia. JAMA 1998;279:1605-6.

2. Safdar N, Dezfulian C, Collard HR, Saint S. Clinical and economic consequences of ventilator-associated pneumonia: A systematic review. Crit Care Med 2005;33:2184-93.

3. Vincent JL, Bihari DJ, Suter PM, et al. The prevalence of nosocomial infection in intensive care units in Europe. Results of the European Prevalence of Infection in Intensive Care (EPIC) Study. EPIC International Advisory Committee.

JAMA 1995;274:639-44.

4. Rello J, Quintana E, Ausina V, et al. Incidence, etiology, and outcome of nosocomial pneumonia in mechanically ventilated patients. Chest 1991;100:439-44.

5. Celis R, Torres A, Gatell JM, Almela M, Rodriguez-Roisin R, Agusti-Vidal A. Nosocomial pneumonia. A multivariate analysis of risk and prognosis. Chest 1988;93:318-24.

6. Craven DE, Kunches LM, Lichtenberg DA, et al. Nosocomial infection and fatality in medical and surgical intensive care unit patients. Arch Intern Med 1988;148:1161-8.

7. Jimenez $\mathrm{P}$, Torres $\mathrm{A}$, Rodriguez-Roisin $\mathrm{R}$, et al. Incidence and etiology of pneumonia acquired during mechanical ventilation. Crit Care Med 1989;17:882-5.

8. Fagon JY, Chastre J, Vuagnat A, Trouillet JL, Novara A, Gibert C. Nosocomial pneumonia and mortality among patients in intensive care units. JAMA 1996;275:866-9.

9. Kerver AJ, Rommes JH, Mevissen-Verhage EA, et al. Colonization and infection in surgical intensive care patients a prospective study. Intensive Care Med 1987;13:347-51. unavailable for analysis; it has been suggested that the Candida species may impact the outcome of patients developing invasive candidiasis (36). The retrospective analysis of the present study does not allow clarification of the question regarding whether RT Candida colonization is a marker of disease or actually contributes to the observed poorer clinical outcomes. The present study included patients with negative bacterial culture CSVAP and does not generalize to patients without such a suspicion. Few patients in each group received antifungal treatment, which was at the clinicians' discretion and could be performed at any time during the 28-day study period. Therefore, the impact of antifungal treatment on patient outcomes and on our results could not be adequately measured within the present study or controlled for as a confounding variable. Finally, the absence of quantitative cultures also limits the generalization of the results.

\section{CONCLUSION}

Our study demonstrated that in patients with a CSVAP with no identifiable bacterial pathogen, the presence of Candida species in RT cultures was associated with higher ICU and hospital mortality, longer time on MV, and an increased ICU and hospital length of stay. Whether Candida species colonization of RT secretions is a marker of disease severity or actually contributes to these poorer clinical outcomes requires further evaluation. The role of antifungal therapy in patients with a CSVAP who grow Candida in their RT secretions also remains to be determined. A randomized trial of antifungal therapy in this specific ICU patient population is currently underway in Canada (www.clinicaltrials.gov, number NCT00934934).

DISCLOSURES: The authors have no competing financial interests to declare. No financial support was received for this secondary analysis. Work for this project was performed at Kingston General Hospital (Kingston, Ontario).

AUTHOR CONTRIBUTIONS: MSD drafted the manuscript and participated in the design of the study. DRW, MA and MMP participated in the design of the study and helped to draft the manuscript. XJ and AGD performed the statistical analysis and participated in the design of the study. DKH conceived the study, participated in the design and coordination, and helped to draft the manuscript. All authors approved the final manuscript

10. Heyland DK, Cook D, Dodek P, Muscedere J, Day A. A randomized trial of diagnostic techniques for ventilator-associated pneumonia. N Engl J Med 2006;355:2619-30.

11. Kollef MH, Morrow LE, Niederman MS, et al. Clinical characteristics and treatment patterns among patients with ventilator-associated pneumonia. Chest 2006;129:1210-8.

12. Luna CM, Vujacich P, Niederman MS, et al. Impact of BAL data on the therapy and outcome of ventilator-associated pneumonia. Chest 1997;111:676-85.

13. Michel F, Franceschini B, Berger P, et al. Early antibiotic treatment for BAL-confirmed ventilator-associated pneumonia: A role for routine endotracheal aspirate cultures. Chest 2005;127:589-97.

14. Muscedere JG, Martin CM, Heyland DK. The impact of ventilator-associated pneumonia on the Canadian health care system. J Crit Care 2008;23:5-10.

15. Leon C, Ruiz-Santana S, Saavedra P, et al. Usefulness of the "Candida score" for discriminating between Candida colonization and invasive candidiasis in non-neutropenic critically ill patients: A prospective multicenter study. Crit Care Med 2009;37:1624-33.

16. Pittet D, Monod M, Suter PM, Frenk E, Auckenthaler R. Candida colonization and subsequent infections in critically ill surgical patients. Ann Surg 1994;220:751-8.

17. Charles PE, Dalle F, Aube H, et al. Candida spp. colonization significance in critically ill medical patients: A prospective study. Intensive Care Med 2005;31:393-400.

18. Eggimann P, Pittet D. Candidiasis among non-neutropenic patients: From colonization to infection. Ann Fr Anesth Reanim 2001;20:382-8. 
19. el-Ebiary M, Torres A, Fabregas N, et al. Significance of the isolation of Candida species from respiratory samples in critically ill, non-neutropenic patients. An immediate postmortem histologic study. Am J Respir Crit Care Med 1997;156:583-90.

20. Wood GC, Mueller EW, Croce MA, Boucher BA, Fabian TC. Candida sp. isolated from bronchoalveolar lavage: Clinical significance in critically ill trauma patients. Intensive Care Med 2006:32:599-603.

21. Meersseman W, Lagrou K, Spriet I, et al. Significance of the isolation of Candida species from airway samples in critically ill patients: A prospective, autopsy study. Intensive Care Med 2009;35:1526-31.

22. Rello J, Esandi ME, Diaz E, Mariscal D, Gallego M, Valles J. The role of Candida sp isolated from bronchoscopic samples in non-neutropenic patients. Chest 1998;114:146-9.

23. Azoulay E, Timsit JF, Tafflet M, et al. Candida colonization of the respiratory tract and subsequent pseudomonas ventilator-associated pneumonia. Chest 2006;129:110-7.

24. Delisle MS, Williamson DR, Perreault MM, Albert M, Jiang X, Heyland DK. The clinical significance of Candida colonization of respiratory tract secretions in critically ill patients. J Crit Care 2008;23:11-7.

25. Heyland DK, Dodek P, Muscedere J, Day A, Cook D. Randomized trial of combination versus monotherapy for the empiric treatment of suspected ventilator-associated pneumonia. Crit Care Med 2008;36:737-44.

26. Harrell FE. Regression modeling strategies: With applications to linear models, logistic regression, and survival analysis. New York: Springer, 2001.

27. Hosmer DW, Lemeshow S. Applied logistic regression. New York: Wiley, 1989.
28. Schoenfeld D. Partial residuals for the proportional hazards regression model. Biometrika 1982;69:239-41.

29. D'Agostino RB Jr. Propensity score methods for bias reduction in the comparison of a treatment to a non-randomized control group. Stat Med 1998;17:2265-81.

30. Olaechea PM, Palomar M, Leon-Gil C, et al. Economic impact of Candida colonization and Candida infection in the critically ill patient. Eur J Clin Microbiol Infect Dis 2004;23:323-30.

31. Young SH, Ostroff GR, Zeidler-Erdely PC, Roberts JR, Antonini JM, Castranova V. A comparison of the pulmonary inflammatory potential of different components of yeast cell wall. J Toxicol Environ Health A 2007;70:1116-24.

32. Roux D, Gaudry S, Dreyfuss D, et al. Candida albicans impairs macrophage function and facilitates Pseudomonas aeruginosa pneumonia in rat. Crit Care Med 2009;37:1062-7.

33. Williamson D, Albert M, Delisle MS, et al. Effect of Candida Spp. in respiratory tract secretions on systemic inflammation. Crit Care Med 2008;36:A115.

34. Nseir S, Jozefowicz E, Cavestri B, et al. Impact of antifungal treatment on Candida-Pseudomonas interaction: A preliminary retrospective case-control study. Intensive Care Med 2007;33:137-42.

35. Leon C, Ruiz-Santana S, Saavedra P, et al. A bedside scoring system ("Candida score") for early antifungal treatment in non-neutropenic critically ill patients with Candida colonization. Crit Care Med 2006;34:730-7.

36. Wisplinghoff H, Bischoff T, Tallent SM, Seifert H, Wenzel RP, Edmond MB. Nosocomial bloodstream infections in US hospitals: Analysis of 24,179 cases from a prospective nationwide surveillance study. Clin Infect Dis 2004;39:309-17. 


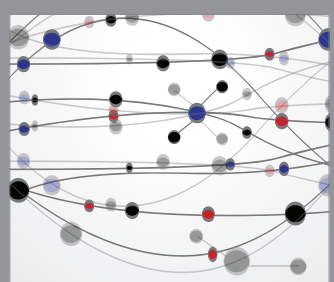

The Scientific World Journal
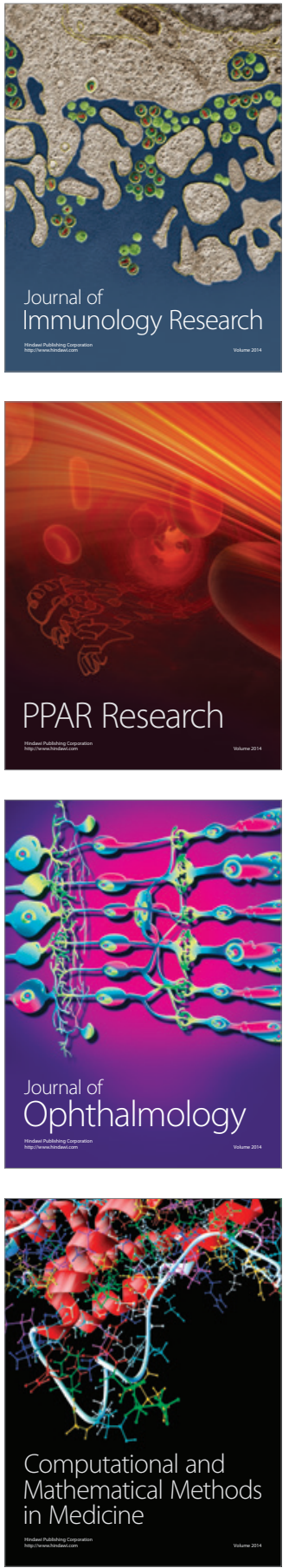

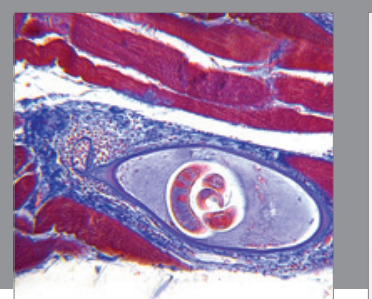

Gastroenterology Research and Practice

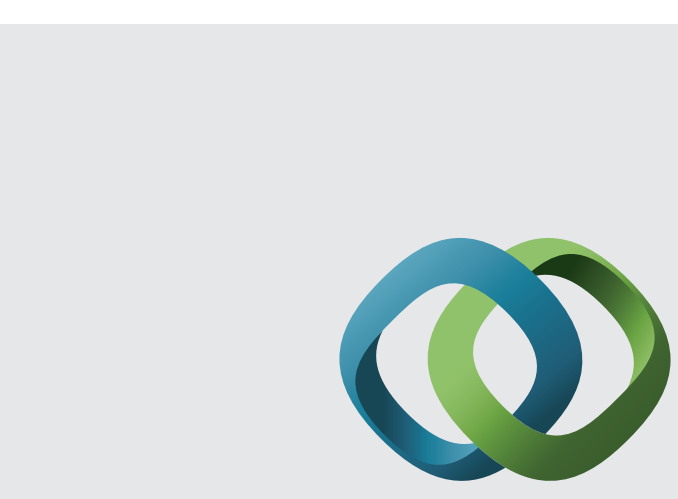

\section{Hindawi}

Submit your manuscripts at

http://www.hindawi.com
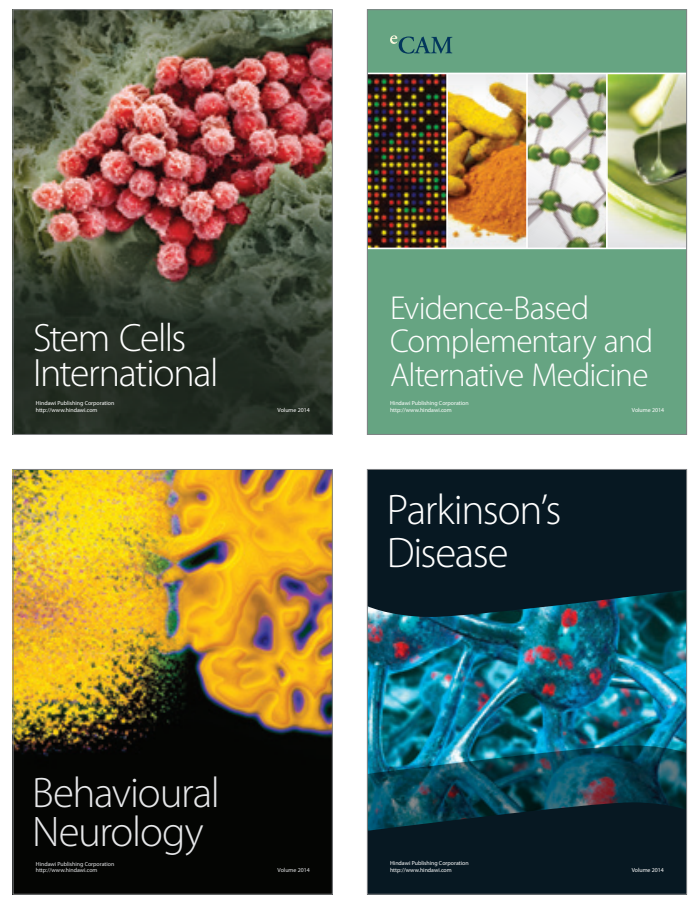
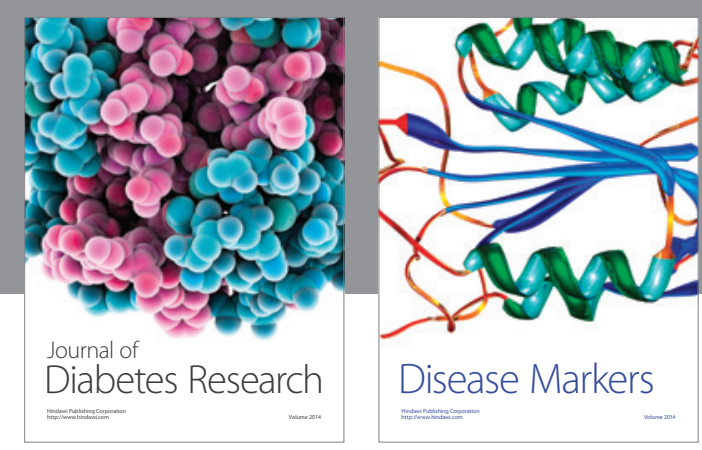

Disease Markers
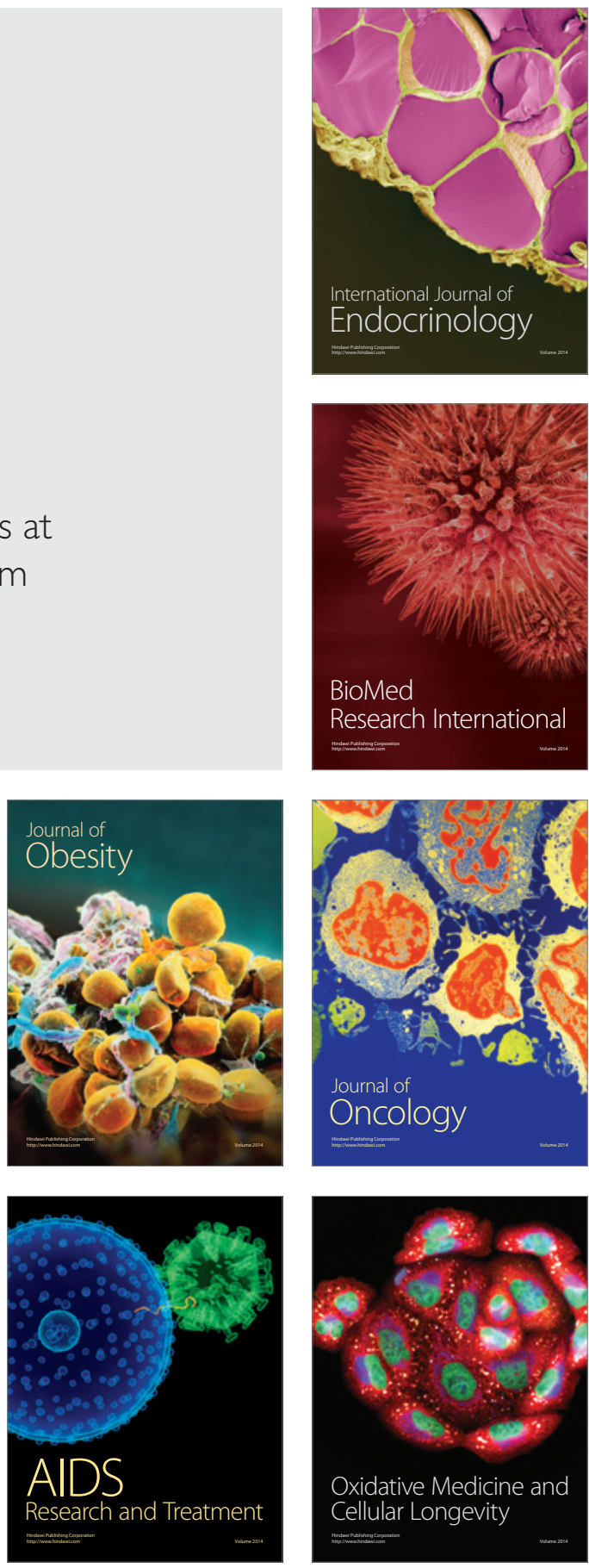